Pteridines

Vol. 9, 1998, pp. $212-216$

\title{
Serum Neopterin Assay to Interpret the Main Cause of Chronic Liver Damage Involved with Plural Etiological Factors
}

\author{
Satomine Yoshimoto ${ }^{1}$, Akira Fukuda ${ }^{1 \S}$, Toshio Mazda ${ }^{2}$ and Nakaaki Osawa ${ }^{1}$ \\ ${ }^{1}$ First Department of Internal Medicine, Osaka Medical College, 2-7, Daigaku-cho, Takatsuki, Osaka \\ 569, Japan \\ ${ }^{2}$ Japanese Red Cross Tokyo Metropolitan Blood Center, 1-26-1, Kyonan-cho, Musashino, Tokyo 180, Japan
}

(Received August 10, 1998)

\section{Summary}

Identification of the main cause of chronic liver damage (CLD) involved with plural etiological factors is important for the management and the treatment of patients. To evaluate its clinical usefulness in interpreting the main cause of CLD involved with hepatitis C virus, alcoholic overdrinking, or fatty steatosis, which are the most frequent and troublesome etiological facotors in the daily outclinic, serum neopterin level was measured in ten healthy subjects, ten chronic hepatitis $\mathrm{C}(\mathrm{CH}-\mathrm{C})$ patients, nine alcohol-induced liver damge (AL) patients, eight non-alcholic fatty liver (FL) patients, eight $\mathrm{CH}-\mathrm{C}$ with AL padtients, seven $\mathrm{CH}-\mathrm{C}$ with $\mathrm{FL}$ patients and ten non-B non-C chronic hepatitis (CH-NBNC) patients by using radioimmunoaasay. There were no significant differences in the mean serum neopterin levels among the healthy subjects, the AL patients and the FL patients. The serum neopterin level in the $\mathrm{CH}-\mathrm{C}$ with $\mathrm{AL}$ or FL patients was significantly higher than that in the AL or the FL patients and just as high as that in the CH-C patients. Furthermore, serum neopterin level in CH-NBNC patients was as high as that in the patients with $\mathrm{CH}-\mathrm{C}$. Therefore, the serum neopterin assay can easily assume the main cause of CLD involved with plural causes, so it will be very useful in the daily clinical practice as an easyto-use auxiliary test.

Key words : Neopterin, Chronic liver damage, Chronic hepatitis C, Alcoholic-induced liver damage, Fatty liver, Chronic non-B non-C hepatitis

\section{Introduction}

In the daily outclinic, if the patients have chronic liver damage (CLD), identification of the main cause of CLD is very important for the management and treatment. The patients with chronic viral hepatitis $(\mathrm{CH})$ have the risks of

\footnotetext{
${ }^{\S}$ Authors to whom correspondence should be addressed. Akira Fukuda, M.D., Osaka Medical College, 2-7, Daigaku-cho, Takatsuki, Osaka 569, Japan
}

infections and may lead to cirrhosis and hepatocellular carcinoma, so appropriate and specific therapy is required $(1,2)$. On the other hand, there are no risks of infections associated with alcohoi-induced liver damage (AL) or nonalcoholic fatty liver (FL), and their prognoses are favorable, because these diseases are mainly treated by modifying the living style, such as abstaining from alcohol or improving diet. Therefore, depending on the etiology of liver injury, management and treatment are drastically

Pteridines $/$ Vol. $9 /$ No. 4 
different, and consequently to develop a therapeutic policy, it is necessary to ascertain the pathology of CLD.

Recently, liver damage caused by alcoholic overdrinking or fatty steatosis due to overfeeding, obesity and diabetes mellitus has been on the rise. Consequently, there are more patients with chronic viral hepatitis complicated with FL or AL. Therefore, when these plural etiological factors are involved, it is often difficult to identify the main cause of CLD, and so a brief test that can easily determine the main cause of liver damage will be very useful in the daily clinical practice.

Of the various liver function tests, alaninamino transferase (ALT) is a sensitive marker to detect liver injury, but this alone is not sufficient to determine the etiology of liver damage. Also, various hepatitis virus markers such as $\mathrm{HBs}$ antigen and anti-HCV antibody are important in screening the cause of liver damage. Nonetheless, it has been shown that some patients are asymptomatic carriers with normal liver functions $(3,4)$. Furthemore, there are some chronic non-B, non-C hepatitis patients who are negative for the above markers. Although liver biopsy is an effective technique to diagnose definitely, it is an invasive procedure, and so it is not appropriate in the daily practice.

Neopterin is a metabolic product of a biosynthesis process in conversion of guanosine 3 phosphate to tetrahydrobiopterin $(5,6)$. This compound is a useful marker that reflects the activated cellular immunity $(7,8)$. There have been several reports on clinical significance of neopterin assay in various liver disease (9-15). Nevertheless, there have not been any reports on the usefulness of serum neopterin assay in ascertaining the main cause of CLD when several etiological factors are involved.

Hepatitis C virus, alcoholic overdrinking, fatty steatosis and hepatitis non- $B$ non- $C$ virus are the most frequent and particularly troublesome etiological factors of CLD in the daily practice in Japan. Therefore, the aim of this study is to investigate the clincal usefulness of serum neopterin assay in interpreting the main cause of CLD involved with these etiological factors.

\section{Subjects and Methods}

\section{Healthy subjects}

Healthy adult blood donors, 14 men and 15 women whose age ranged from 20 to 65 years with a mean age of 49 years, were randomly selected as healthy subjects. The mean level of serum neopterin was $4.3 \pm 0.7 \mathrm{nmol} / \mathrm{L}$ for the men and $4.4 \pm 0.4 \mathrm{nmol} / \mathrm{L}$ for the women, respectively. There were no significant differences in the mean serum neopterin concentration attributable to sex or age, thus suggesting that the level of serum neopterin dose not fluctuate between the sexes within this age range. Accordingly, ten healthy subjects $(6$ men and 4 women) of age matching to that of the CLD patients were randomly chose for comparison.

\section{Patients}

We studied sixty patients with CLD, including ten patients with chronic hepatitis $\mathrm{C}$ ( $\mathrm{CH}-\mathrm{C})$, nine patients with alcohol-induced liver damge (AL), eight patients with non-alcoholic fatty liver (FL), eight patients with chronic hepatitis $\mathrm{C}$ complicated with alcoholic-induced lver damage (CH-C with AL), seven patients with chronic hepatitis $\mathrm{C}$ complicated with non-alcoholic fatty liver ( $\mathrm{CH}-\mathrm{C}$ with FL) and ten patients with non$B$ non-C chronic hepatitis (CH-NBNC).

All patients had abnormal liver function for at least six months. The main cause of chronic liver damage in these patients could not be clarified by only their routine liver function tests. The diagnosis of chronic hepatitis $\mathrm{C}$ was based on the presence of serum anti-HCV (II) antibody (Ortho Diagnostics, ELISA) and HCV-RNA by the RT-PCR assay, the histological findings of liver biopsy samples and the absence of clinical, histological or serological signs of other viral, toxic, metabolic or autoimmune hepatitis. The AL patients had the long history of alcoholic abuse and confirmed histologically showing either fatty change or alcoholic fibrosis. In all $\mathrm{AL}$ patients, their liver function markedly improved after stop drinking. The FL patients were diagnosed by ultrasonographic and histological findings. These patients had associated disease such as overfeeding, obesity or hyperlipidemia. Both the AL patients and the FL patients were negative to clinical or serological sign of other causes of liver diseases. The chronic hepatitis $C$ patients complicated with $\mathrm{AL}$ or FL had both clinical or histological findings of chronic viral hepatitis and alcoholic-induced liver injury or nonalcoholic fatty steatosis. The patients with non-B non-C hepatitis were all negative for anti-HCV antibody, HBs antigen and other signs of liver diseases, but they were diagnosed histologically as 
chronic viral hepatitis.

\section{Methods}

Blood samples were collected at the same time of performing the liver function tests, and the serum was immediately separated. They were then stored frozen at $-30^{\circ} \mathrm{C}$ until the concentration of neopterin was ready to be measured.

The routine liver function tests were performed using a chemical autoanalyzer (Hitachi 760-60E, Tokyo, Japan). The hepatitis virus markersn was detected by using widely available commercial kits. The serum neopterin level (nmol/L) was assayed by using an RIA kit (IMMU test neopterin, Henning Berlin GMBH, Berlin, GER) (16), and its radioactivity was measured using a gamma counter (ANSR, Dinabbot Co., Ltd., Tokyo, Japan). All data are expressed as mean $\pm S D$.

The differences among healthy subjects and patients groups were statistically analyzed by the Mann-Whitney U-test, and $\mathrm{p}<0.05$ was considered significant.

\section{Results}

As shown in Table 1 , there were no significant diffeences in age or ALT among the different groups. Also, although the level of gamma glutamyl transpeptidase (GGT) in the AL patients and in the $\mathrm{CH}-\mathrm{C}$ with $\mathrm{AL}$ patients were higher than those in the other groups, there were no significant differences between the two groups. Consequently, the routine liver function tests failed to clarify whether hepatitis $\mathrm{C}$ virus, alcohol abuse, of fatty steatosis was the main cause of liver damage among patients with plural etilogical factors.
Table 2. Serum neopterin level in healthy subjects and patients with CLD

\begin{tabular}{lc}
\hline \hline & neopterin* $(\mathrm{nmol} / \mathrm{L})$ \\
\hline Healthy subjects & $4.4 \pm 0.6$ \\
Patients with CLD & $4.4 \pm 0.9$ \\
FL & $4.2 \pm 0.6$ \\
AL & $8.2 \pm 2.3$ \\
CH-C & $8.2 \pm 2.8$ \\
CH-C with FL & $7.1 \pm 1.2$ \\
CH-C with AL & $8.8 \pm 2.2$ \\
CH-NBNC &
\end{tabular}

\section{${ }^{*}$ Mean \pm SD.}

p; Healthy subjects vs FL, AL: not significant.

FL vs CH-C with FL: $<0.001$

$\mathrm{AL}$ vs $\mathrm{CH}-\mathrm{C}$ with $\mathrm{AL}:<0.001$

CH-C vs $\mathrm{CH}-\mathrm{C}$ with $\mathrm{FL}$, $\mathrm{CH}-\mathrm{C}$ with AL: not significant.

Table 2 shows the mean serum level of neopterin in the healthy subjects and the CLD patients. The mean neopterin level in the healthy men and women was $4.5 \pm 0.2 \mathrm{nmol} / \mathrm{L}$ and $4.3 \pm$ $1.0 \mathrm{nmol} / \mathrm{L}$, respectively. These results agree with other previous report as the normal values of serum neopterin, and there was no significant difference between the sexes (17).

The serum neopterin level in the $\mathrm{CH}-\mathrm{C}$ with AL patients was significantly higher than that in the AL patients. Also, the serum neopterin level in the CH-C with FL patients was higher than that in the FL patients. The serum neopterin level in the $\mathrm{CH}-\mathrm{C}$ patients was just as high as that in the $\mathrm{CH}-\mathrm{C}$ with $\mathrm{AL}$ or $\mathrm{FL}$ patients, and there were no significant differences in the serum levels of neopterin among these patients groups. Furthermore, there were no significant differences in the serum neopterin level among the healthy individuals, the AL patients and the FL patients. Therefore, the serum concentration of neopterin was significantly high in the patients groups

Table 1. Profiles of healthy subjects and patients with chronic liver disease

\begin{tabular}{lrccc}
\hline \hline & $\mathrm{n}(\mathrm{M} / \mathrm{F})$ & Age $(\text { years })^{*}$ & ALT $(\mathrm{U} / \mathrm{l})^{*}$ & $\mathrm{GGT}(\mathrm{U} / \mathrm{l})^{*}$ \\
\hline Healthy subjects & $10(6 / 4)$ & $56 \pm 5.4(47-62)$ & $19 \pm 9.3$ & $\mathrm{ND}^{\dagger}$ \\
Patients with CLD & $8(4 / 4)$ & $51 \pm 8.7(39-68)$ & $82 \pm 31$ & $85 \pm 49$ \\
FL & $9(8 / 1)$ & $52 \pm 6.4(44-60)$ & $94 \pm 84$ & $141 \pm 164^{\ddagger}$ \\
AL & $10(3 / 7)$ & $53 \pm 8.8(37-61)$ & $124 \pm 67$ & $84 \pm 90$ \\
CH-C & $7(3 / 4)$ & $57 \pm 5.9(49-66)$ & $94 \pm 64$ & $74 \pm 69$ \\
CH-C with FL & $8(7 / 1)$ & $55 \pm 6.8(47-65)$ & $108 \pm 96$ & $190 \pm 133^{\ddagger}$ \\
CH-C with AL & $10(5 / 5)$ & $57 \pm 7.5(41-65)$ & $113 \pm 53$ & $72 \pm 34$ \\
CH-NBNC & & & \\
\hline
\end{tabular}

CLD, chronic liver disease; FL, non-alcoholic fatty liver

$\mathrm{AL}$, alcohol-induced liver disease

$\mathrm{CH}-\mathrm{C}$, chronic hepatitis $\mathrm{C}$; $\mathrm{CH}-\mathrm{NBNC}$, charonic hepatits non-B non-C

ALT, alanine aminotransferase; GGT, gamma glutamyl transpeptidase.

${ }^{*}$ Mean \pm SD. ${ }^{\dagger}$ not done.

${ }^{\ddagger} \mathrm{p}$; AL, CH-C with AL vs FL, CH-C, CH-C with FL, CH-NBNC: <0.01. AL vs CH-AL: not significant.

Pteridines /Vol. $9 /$ No. 4 


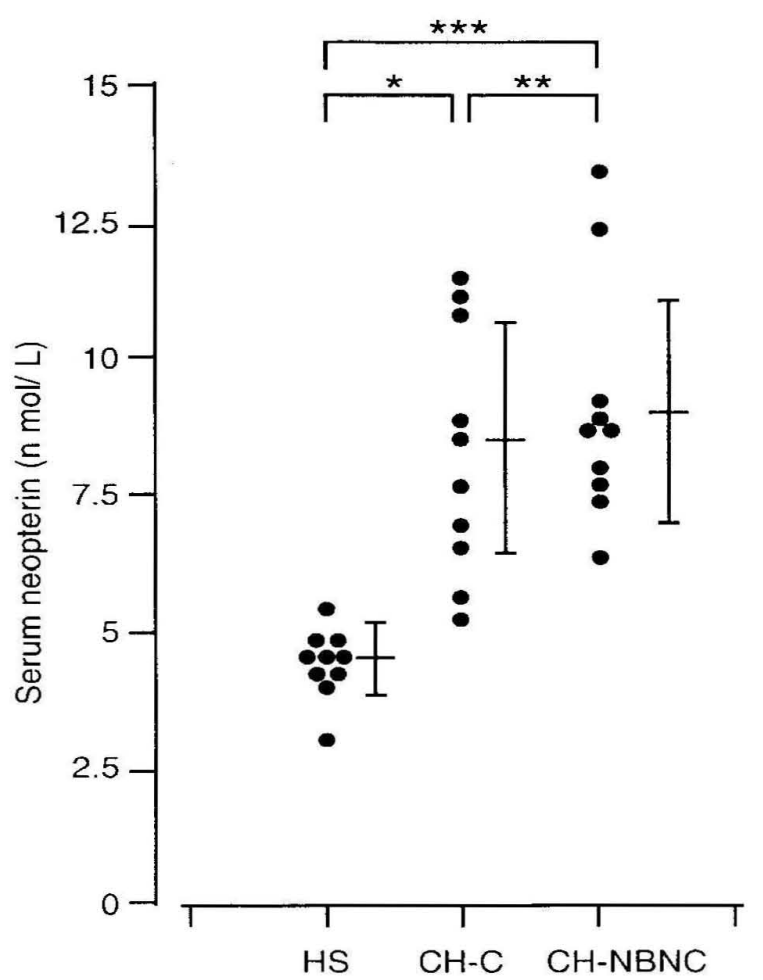

Figure 1. Serum neopterin level in healthy subjects (HS), chronic hepatitis $\mathrm{C}$ patients $(\mathrm{CH}-\mathrm{C})$ patients and chronic non-A non-B hepatitis patients (CH-NBNC). Serum neopterin values $(\mathrm{nmol} / \mathrm{L})$ for each healthy subject and patient are plotted $(\bullet)$ and the mean $\pm S D$ for each group is also given. ${ }^{\star} \mathrm{p}<0.001 ;{ }^{\star \star} \mathrm{p}$ : not significant.; ${ }^{\star \star \star}$ $\mathrm{p}<0.001$.

complicated with $\mathrm{CH}-\mathrm{C}$ regardless of factors such as alcohol abuse $r$ fatty steatosis.

In addition, the serum neopterin levels in the CH-NBNC patients was just as high as that in the patients with CH-C. Moreover, serum neopterin levels of all CH-NBNC patients were higher than those of healthy subjects (Figure 1).

\section{Discussion}

The results of the presednt study showed that measurement of serum neopterin level would be useful in determining whether hepatitis $C$ virus was the main cause of CLD among those patients with plural etiological factors, such as hepatitis $\mathrm{C}$ virus, alcoholic abuse, or fatty steatosis.

The etiology of CI.D greatly varies. In Japan, due to its incidence and the problems associated with its prognosis, hepatitis $C$ virus is the most important etiological factor for CLD. Furthermore, the number of AL or FL patients caused by drinking, overfeeding or obesity is recently increasing, and they are most frequently observed in the daily outclinic. Accordingly e are now many patients with mixed etilogical factors such as hepatitis C virus, alcohlic overdrinking, and fatty liver. In these patients, the serum neopterin assay can easily provide additional information in determining the main cause of chronic liver damage. In other words, if the level of serum neopterin is high, then we can deduce that hepatitis $\mathrm{C}$ virus is likely to the main cause of liver damage, but if the level of serum neopterin is within the normal range, then we can assume that overdrinking or fatty liver is the main cause of liver damage.

In the diagnosis of $\mathrm{AL}$, one of the clinically important findings is that the liver dysfunction is improved by abstaining from alcoholic drinking. Nontherless, in then outpatient clinics, it is generally difficult to order the AL patients who consume large quantities of alcohol to abstsin from drinking. Therefore, it has often been difficult to determine the main cause of liver damage among patients with both hepatitis $C$ virus and overdrinking history. The results of the present study showed that if the serum neopterin level is high, then hepatitis $\mathrm{C}$ virus is more likely to be the main cause of liver damage.

Due to recent advances in diagnostic imaging, FL can be non-invasively and easily diagnsed by abdominal ultrasonography. Nontheless, the presence of fatty liver is not necessarily accompanied by liver dysfunction, and it takes a long time to improve fatty deposit by diet or exercise treatment. Therefore, it has been difficult to determine the main cause of its liver damage in the patients with both hepatitis $\mathrm{C}$ virus and fatty liver. Also in these patients, if the serum neopterin level is within the normal range, then we can deduce that fatty liver is the main cause, and if the serum neopterin level is high, then we can assume that hepatitis $C$ virus is the main cause of its liver damage.

There are several reports that the level of neopterin increases in the patients with alcoholinduced liver injury $(18,19)$. In the present study, however, the serum neopterin level did not significantly increase in the patients who consumed large quantities of alcohol. This may be partially explained by the fact that the degree of liver injury caused by alcohol consumption markedly varies.

The increase in neopterin correlates with the activation of macrophages via $\gamma$-IFN stimulation (20), and this increase is generally thought to reflect an acceleration in the cellular immunity (8, 
14). Based on the mechanism of increase in the neopterin level, it is likely that liver injury due to hepatitis C may more closely correlated with the immune system than that due to alcohol or fatty change.

In the present study, the serum neopterin level was relatively high in the $\mathrm{CH}-\mathrm{NBNC}$ patients and in the CH-C patients. This findings shows that even in the absence of obvious etiological factors, the elevated serum neopterin level suggests the existence of unknown types of hepatitis viral infection (13). Moreover, neopterin assay may also be used to screen blood donors to prevent the post-transfusion hepatitis caused by unknown hepatitis virus which there are no viral markers at present.

Terefore, the serum neopterin assay would be useful as an easy-to-use auxiliary test in the outpatient clinics since the immunological status of the patients can be assessed simultaneously by using blood samples.

\section{References}

1. Di Bisceglie AM, Rustgi VK, Hoofnagle JH, Dusheiko GM, Lotze MT; Hepatocellular carcinoma. Ann Intern Med 1998; 108: 390-401.

2. Ruiz J, Sangro B, Cuende JI, et al. Hepatitis B and $C$ viral infections in patients with hepatocellural carcinoma. Hepatology 1992; 16: 637-641.

3. Zanetti AR, Tanzi E, Zehender G, et al. Hepatitis C virus RNA in symptomless donors implicated in posttransfusion non-A, non-B hepatitis. Lancet 1990; 336: 448 .

4. Brillanti, S, Gaiani S, Miglioli M, et al. Persistent hepatitis C viraemia without liver disease. Lancet 1993; 341: 464-465.

5. Fukushima K, Eto I, Mayumi T et al. Biosynthesis of pterines in mammalian systems. In: Pfleder W, ed. Chhemistry and Biology of Pteridines. Berlin-New York: Walter de Gruyter, 1975; 247-263.

6. Muller MM, Curtius HC, Herold M, Huber CH. Neopterin in clinical practice. Clin Chem Acta 1991; 201: 1-6.

7. Wachter H, Fuchs D, Hausen A, Reibengger G, Werner ER. Neopterin as marker for activation of cellular immunity; immunological basis and clinical application. Adv Clin Chem 1989; 27: 81-141.

8. Fuchs D, Hausen A, Reibnegger G, Werner ER, Di- erich MP, Wachter H. Neopterin as a marker for activated cell-mmediated immunity; application in HIV infection. Immunol Today 1988; 9: 150-155.

9. Prior $\mathrm{CH}$, Fuchs D, Hausen A, et al. Potential of urinary neopterin excretion in dfferentiating chronic non-A, non-B hepatitis from fatty liver. Lancet 1987; 11: 1235-37.

10. Reibnegger G, Auhuber I, Fuchs D, Hause A, Judmaier G, Prior C, Werner ER, Wachter H. Urinary neopterin levels in acute viral hepatitis. Hepatology 1988; 8: 771-774.

11. Fukuda A, Mazda T, Gyure WL et al. A comparative study of urinery xanthopterin and neopterin in liver disease. Eur $\mathrm{J}$ Clin Chem Clin Biochem 1993; 31: 129-134.

12. Mazda T, Iino T, Tsusue $M$. Urinary and serum pteridines in liver diseases. Pteridines 1996; 7: 160165.

13. Reibnegger G, Fuchs D, Hausen A, Werner Felmayer, G, Wachter H. Neopterin and viral infections; diagnostic potential in virally induced liver dsease. Biomed Pharmacother 1989; 43: 287-293.

14. Mazda T, Nojiri N, Kimura H, Ino T, Ohbayashi A. Plasma neopterin in heatitis $\mathrm{C}$ virus antibody-positive and -negative donors with elevated alanine aminotransferase activity. Pteridines 1994; 5: 111-113.

15. Wilmer A, Nolchen B, Tilg $\mathrm{H}$ et al. Serum neopterin concentrations in chronic liver disease. Gut 1995; 37: 108-112.

16. Rokos H, Rokos A. A radioimmunoassay for determination of neopterin. In: Blair JA, ed. Chemistry and Biology of Pteridines. Berlin-New York: Walter de Gruyter, 1983; 815-819.

17. Werner ER, Bichler A, Daxenbichler G, et al. Determination of neopterin in serum and urine. Clin Chem 1987; 33: 62-66.

18. Gonzales RE, Santolaria FF, Rodriguez RE, et al. Serum neopterin levels in alcoholic liver disease. Drug-Alcohol-Depend 1993; 33: 151-156.

19. Diez-Ruiz A, Tiltz GP, Gutierrez-Gea F, et al. Neopterin and soluble tumor necrosis factor receptor type $I$ in alcohol-induced cirrhosis. Hepatology $1995 ; 21$ : 976-978.

20. Huber C, Batchelor JR, Fuchs D, et al. Immune response-associated production of neopterin. Release from macrophages primarily under control of interferon gamma. J Exp Med 1984; 160: 310-316.

21. Huber C, Fuchs D, Hausen A, et al. Pteridins as a new marker to detect human $\mathrm{T}$ cells activated by allogeneic or modified self major histocompatibility complex (MHC) determinants. J Immunol 1983; 130: 1047-1050. 